\title{
ALGUNAS OBSERVACIONES SOBRE LA DESAPARICIÓN DEL FUTURO DE SUBJUNTIVO Y SU FORMA COMPUESTA DEL SISTEMA TEMPORAL CASTELLANO
}

\begin{abstract}
Resumen. El presente artículo gira en torno a la cronología de la desaparición de las formas cantare y hubiere cantado del sistema temporal castellano, atendiendo a las diferencias entre el español peninsular y su variedad americana, en las que se fijó en su día Germán de Granda (1978). Debido a la escasez de datos acerca de la forma compuesta del futuro de subjuntivo, el autor realiza un pequeño análisis de su empleo en las obras de la narrativa española de los siglos XVI-XIX recopiladas en el corpus CORDE.
\end{abstract}

Palabras clave: futuro de subjuntivo, antefuturo de subjuntivo, sistema temporal español, posterioridad.

\section{Introducción}

Como ya se ha resaltado en múltiples ocasiones, tanto el futuro de subjuntivo (cantare), así como el antefuturo de subjuntivo (hubiere cantado), según la nomenclatura de Rojo y Veiga (1999), se utilizan hoy en día con muy poca frecuencia, sobreviviendo ante todo en los textos jurídicos, lenguajes técnicos y jergas profesionales especialmente conservadoras (Camús Bergareche, 1990: 411). Además, las formas en cuestión aparecen «en algunas construcciones fijas tipo sea lo que fuere o en estilos deliberadamente solemnes o arcaizantes, donde no es infrecuente encontrar sus usos erróneos, lo que prueba su afuncionalidad en la lengua moderna» (Rojo y Veiga, 1999: 2922).

Recordemos que cantare, al carecer de autosuficiencia sintáctica, empieza a convertirse, sobre todo entre los representantes de la clase social más baja, en un lujo del sistema a principios del siglo XVI (Luquet, 1988: 514; Camús Bergareche, 1990: 421; Nowikow, 1993: 115), pero los indicios

\footnotetext{
* Uniwersytet Łódzki.
} 
de su debilidad se recogen ya en el siglo XIII (López Rivera, 1994: 82-83). Sin embargo, hay que ser cauteloso a la hora de pensar en una rápida eliminación de este tiempo a partir de este momento. En opinión de Herrero Ruiz de Loizaga (2006: 944), «su uso seguirá manteniéndose con fuerza a lo largo del XVII e incluso del XVIII, y probablemente, no solo como recurso de la lengua escrita, sino con un correlato, aunque no tan amplio como en épocas anteriores, en el registro hablado culto». En cuanto a la forma compuesta (hubiere cantado), Penny (2014: 247) pone de manifiesto que «ha compartido la suerte de su equivalente simple, limitándose cada vez más al estilo legal y desapareciendo del uso habitual después del siglo XVIII».

\section{Desaparición del futuro del subjuntivo en el español de América}

Al tratar la caída en desuso del futuro de subjuntivo, hemos de tener muy en cuenta las diferencias entre el español peninsular y su variedad americana, puesto que el trayecto evolutivo del futuro de subjuntivo en la Península ha sido algo diferente al de América, problema en el que que centró su atención Granda (1978). Este gran lingüista advierte que cantare sigue utilizándose con mayor o menor vitalidad en el siglo XX en el español atlántico, es decir, en «un área relativamente compacta y homogénea que, dejando aparte la zona o zonas canarias, abarca las islas hispanohablantes del Caribe (Santo Domingo, Puerto Rico y Cuba) y las costas atlánticas de América del Sur desde Panamá al oeste hasta Venezuela al este» (Granda, 1978: 99).

Sin ir más lejos, es de subrayar que Granda (1978: 106-113) introduce de este modo una nueva repartición diatópica de los hechos según la cual habría que asociar el uso de la forma en -re en dichas regiones con el hecho de que entre los años 1492 y 1530, a saber, durante la llamada «fase antillana» de la conquista, las flotas zarpaban normalmente de Santo Domingo, mientras que las expediciones posteriores partían normalmente de Sevilla. Granda (1978: 106) sugiere que las zonas americanas donde es utilizado el futuro de subjuntivo corresponden a la colonización más antigua («periodo antillano») frente a las que no lo conservan. El foco lingüístico de aquel periodo se encuentra en Santo Domingo y refleja el uso de la lengua en la metrópoli a finales del siglo XV.

En cambio, a Eberenz (1990: 406) le convence mucho más la hipótesis rechazada por Granda «según la cual las zonas en cuestión representarían islotes lingüísticos conservadores donde el futuro de subjuntivo se habría 
mantenido gracias a los escasos contactos con otras regiones». A su modo de ver, al principio cantare también se empleaba en el resto del continente americano para pasar extinguirse probablemente en el siglo XVII. Por otro lado, como señala el lingüista suizo, existe una serie de indicios de que esta evolución fue incluso más lenta en América que en Europa, destacando su uso relativamente frecuente en las obras de Carrió de la Vandera (1715-1783) y en la prosa hispanoamericana de los siglos XIX y XX1 (Eberenz, 1990: 406).

Sin pronunciarnos a favor o en contra de una de las hipótesis, es evidente que durante la época colonial en el continente americano se distinguían con claridad variedades dialectales conservadoras e innovadoras (Fontanella de Weinberg, 1992). Cabe destacar que la existencia de esas dos variedades suscitó una extensa polémica en el siglo $X X$, en la que intervinieron tales lingüistas de reconocido prestigio como Amado Alonso y Pedro Henríquez Ureña, por una parte, y Rafael Lapesa, Diego Catalán y Ramón Menéndez Pidal, por otra, quienes intentaron determinar el peso que la influencia del español de Andalucía tuvo sobre el español de América.

En lo tocante al uso del futuro de subjuntivo en América, consideramos oportuno empezar por las investigaciones llevadas a cabo por Fontanella de Weinberg (1987: 71) en Buenos Aires, Tucumán (Fontanella de Weinberg, 1992: 362) y en Corrientes (Fontanella de Weinberg, 1992: 363) donde se registra con frecuencia la forma de cantare en los siglos XVII y XVIII no solo en el estilo notarial, sino en todo tipo de documentos (Fontanella de Weinberg, 1992: 370).

Ramírez Luengo (2002: 314), a su vez, después de analizar el empleo de cantare en la Banda Oriental, es decir, el territorio ubicado al este del río Uruguay y al norte del Río de la Plata, sobre la costa atlántica de Sudamérica, constata que en esa región del continente americano dicho tiempo permanece vivo en el siglo XVIII tanto en documentos oficinales como en cartas familiares. A pesar de que está presente en todos los estratos sociolingüísticos, es mucho más abundante en los textos pertinentes al registro culto ( $89 \%$ de las ocasiones). Dicho de otro modo, la situación parece muy semejante a la que esboza Fontanella de Weinberg (1992: 370) para el español bonaerense de la misma época, pero debido al carácter más conservador del español de la Banda Oriental en comparación con la «banda occidental», a saber, con Buenos Aires, el proceso de reemplazo del futuro de subjuntivo por otras formas del sistema verbal se intensifica a lo largo del siglo XIX.

${ }^{1}$ Es de subrayar que ni Vicente Salvá, ni Andrés Bello clasifican este tiempo como anticuado en sus gramáticas (Eberenz, 1990: 407). 
Por lo que respecta a la eliminación del futuro de subjuntivo del sistema temporal castellano, cabe mencionar otra contribución de Ramírez Luengo (2008) en la que se comenta su uso en el español centroamericano, aprovechando un conjunto de 19 documentos datados entre 1703 y 1758, y procedentes de la antigua Audiencia de Guatemala, esto es, el territorio que actualmente corresponde a Guatemala, El Salvador, Honduras y Nicaragua. A la luz de los resultados obtenidos, se desprende que cantare mantiene su vitalidad en el siglo XVIII no solo en textos notariales, sino también en cartas privadas de carácter culto-estándar, constituyendo el 16,8\% del total de las formas del modo subjuntivo. Aun así, no hay que perder de vista que un estudio detallado de la distribución sintáctica permite observar que el empleo de futuro de subjuntivo en Centroamérica en el siglo XVIII queda restringido casi exclusivamente a la oración de relativo $(92,10 \%)$, lo cual supone cierto indicio de la debilidad de dicho tiempo.

Asimismo, es indispensable llamar la atención sobre las observaciones de Ruiz de Loizaga (2006: 945, 949), quien documenta la forma cantare en el siglo XVIII en las obras de Antonio Zamora, Ramón de la Cruz, Torres Villarroel y Cañizares. Como apunta Ruiz de Loizaga (2006: 951-952), en la segunda mitad del siglo XVI observamos un retroceso de su empleo, aun distanciado mucho de haberse convertido todavía en forma marginal. Dicho retroceso no se intensifica ni en el siglo XVII, ni incluso en la primera mitad del XVIII. Es en la segunda mitad del siglo XVIII cuando se registra una significante disminución en el uso de futuro de subjuntivo que acaba siendo un tiempo residual a principios del siglo XIX. En cambio, en la segunda mitad del siglo XIX se notan cada vez más los falsos usos de cantare como pretendido equivalente de cantara / cantase, formas con las que nunca había compartido ningún empleo modo-temporal en la historia de la lengua (Rojo y Veiga, 1999: 2922). Si efectivamente es así, entonces no habría una gran diferencia en la cronología de la progresiva eliminación de cantare entre el español peninsular y el de América.

\section{Uso del antefuturo de subjuntivo}

Dado que en los estudios acerca del futuro de subjuntivo se dedica relativamente poca atención al antefuturo de subjuntivo ${ }^{2}$, hemos de resaltar que históricamente el tiempo en cuestión, al igual que el llamado

${ }^{2}$ La misma observación concierne al número de estudios dedicados a las formas cantará y habrá cantado. 
condicional perfecto (avría cantado, sería venido), carecía de antecedentes latinos y se creó como equivalente del paradigma del futuro de subjuntivo: HABERE HABUERO / HABUERIM CANTATUM > ovier(e) cantado > hubiere cantado, etc. (Penny, 2014: 194).

Como advierten Folgar y Ramos (1992: 408), la forma compuesta del futuro de subjuntivo era mucho menos habitual que la simple ya en la Edad Media y esa tendencia se mantuvo en los siglos siguientes. Por eso, no sorprende mucho el hecho de que Ramírez Luengo (2002: 309), al analizar el corpus compuesto por 36 documentos (19 textos legales y 17 cartas familiares) del siglo XVIII escritos en la Banda Oriental, sobre todo en Montevideo, solo consiguiera encontrar un ejemplo de hubiere cantado (1,78\% del total) frente a los 55 ejemplos de cantare (98,21\%).

Desde el punto de vista sintáctico, el antefuturo ocupaba una posición privilegiada ${ }^{3}$ en oraciones relativas con pronombre que y con antecedente expreso determinado. El determinante aparecía como primer elemento de una frase nominal o precedía directamente a la oración relativa:

...conuiene que tales obras fagan por que con derecho acresçienten su regno et fagan en guisa que en su vida sean muy loados de las gentes, et despues de su muerte finquen buenas fazannas de las buenas obras ellos ovieren fechas (Lucanor, 41, 30, apud Folgar y Ramos, 1992: 415)

... a las que fueren costunbradas de comer dos uezes al dja çéuenlas a la ora de las biésperas (Cetrería, 14, 8, apud Volgar y Ramos, 1992: 416)

Amén de ello, hubiere cantado se empleaba con frecuencia en oraciones subordinadas adverbiales introducidas por algunas de las siguientes conjunciones o locuciones conjuntivas: como, después que, desque, pues que, quando y si:

...estos vuestros consejeros vos lo dizen por que saben que desque en tal fecho vos ovieren metido, que por fuerça abredes a fazer lo que ellos quisieren (Lucanor, 4, 49, apud Volgar y Ramos, 1992: 416)

...conuiene que cada vn aue commo lo ouiere vsado que asy le fagan (Cetrería, 19, 56, apud Volgar y Ramos, 1992: 416)

(5) demás, el precio todo cuando fuere llegado para cerca de villa quiero que seya dado (Apolonio, 87c, apud Volgar y Ramos, 1992: 416)

De acuerdo con el valor que le corresponde, el antefuturo de subjuntivo denota una situación contingente anterior a otra que es posterior a algún

${ }^{3}$ Como se señala en la NGLE (2009: 1815), hubiere cantado se utilizaba en los mismos contextos que cantare, a saber, era empleado ante todo en prótasis condicionales y concesivas, oraciones de relativo y como término de algunas conjunciones subordinantes. 
punto de referencia (NGLE, 2009: 1812-1813). Así, hubiere cantado se refiere a una situación hipotética en el fragmento siguiente:

(6) Si dentro de treinta días a partir de la fecha de inscripción del registro del jugador, los clubes no hubieren llegado a un acuerdo sobre el monto de la indemnización, el Comité Ejecutivo tiene facultad para establecer el monto de la indemnización (ABC Color 17.09.1996, apud NGLE, 2009: 1814).

Es obvio que la prótasis condicional aporta en este caso el contexto modal (Si dentro de treinta días...). El texto informa, por tanto, de que algo ocurrirá si la consecución de cierto acuerdo no tiene lugar en un momento anterior al final de un lapso de treinta días que se cuentan desde el momento en que se realiza determinada inscripción.

Hoy en día, la forma compuesta del futuro de subjuntivo es utilizada en los textos jurídicos con el fin de atenuar el carácter hipotético de la situación que se describe. Sin embargo, desde hace tiempo queda reemplazada con frecuencia por hubiera cantado, tanto en el sentido en el que hace referencia a lo venidero como en el que alude a una situación pasada que se considera improbable:

(7) En el caso de que se hubieren producido esas circunstancias, entonces el proyecto histórico que se propuso escribir, habría sido notoriamente acertado (Magallón, Renacimiento, apud NGLE, 2009: 1815).

Por último, el antefuturo de subjuntivo puede expresar probabilidad con respecto al presente o antepresente. De este modo, hubiere venido admite la paráfrasis «puede (ser) que haya venido» en el texto siguiente:

(8) Yo creo que la vida no debe ser una meditación sobre la muerte sino una meditación sobre la vida, y solo me retiene a su lado la gente que mira las cosas, aun las más insignificantes, aun las que parecen invitar menos a la meditación, con la actitud de quien hubiere venido desde una región noble a este planeta para hacer una compulsa sincera (Mallea, Bahía, apud NGLE, 2009: 1815).

\section{Desaparición del antefuturo de subjuntivo del español peninsular}

A nuestro juicio, el uso relativamente reducido de hubiere cantado a lo largo de su presencia en castellano puede dificultar la posibilidad de indicar el momento de su desaparición del sistema temporal. Como ya hemos dicho, Penny (2014: 247) señala que la forma en cuestión deja 
de utilizarse después del siglo XVIII sin distinguir entre el español peninsular y su variedad americana. Sea como fuere, a continuación, vamos a presentar los resultados de nuestro pequeño análisis basado en el corpus CORDE, que abarca el empleo de la forma en cuestión en la narrativa española de los siglos XVI-XIX. Hemos elegido la narrativa, ya que creemos que refleja bien las tendencias presentes en la lengua hablada de la época que representa. Desgraciadamente, el análisis del uso de hubiere cantado en cualquier país de Sudamérica resultó imposible a causa de la falta de ejemplos recopilados en el corpus.

Tabla 1. El uso del antefuturo de subjuntivo en la narrativa española de los siglos XVI-XIX

\begin{tabular}{|c|c|}
\hline Siglo & Número de casos \\
\hline XVI & $14^{4}$ \\
\hline XVII & 58 \\
\hline XVIII & 4 \\
\hline XIX & 9 \\
\hline
\end{tabular}

Fuente: elaboración propia.

Tal y como se ve, parece que el antefuturo de subjuntivo cae en desuso en el español peninsular hacia el siglo XVIII. Aunque no disponemos de suficientes datos para señalar con mayor precisión cuándo empieza dicho proceso, es indudable que hubiere cantado todavía se emplea en repetidas ocasiones en el siglo XVII. Lo usan en sus obras, entre otros, Mateo Alemán, Miguel de Cervantes, Lope de Vega, Francisco de Quevedo y Villegas, y Juan de Zabaleta. La supuesta desaparición de hubiere cantado en el siglo XVIII no difiere mucho de la cronología de cantare, lo cual nos hace suponer que el antefuturo de subjuntivo también se utilizaba poco en aquella época en el continente americano.

\section{Observaciones finales}

Recapitulando todo lo dicho hasta aquí, podemos extraer las siguientes conclusiones con respecto a la eliminación de los futuros de subjuntivo del sistema temporal castellano:

${ }^{4}$ La escasez de ejemplos encontrados para el siglo XVI probablemente está vinculada con el número reducido de los textos de aquella época recogidos en el corpus. 
a) el futuro de subjuntivo deja de utilizarse en España casi por completo en el siglo XIX, pero el retroceso de su empleo se produce hacia la mitad del siglo XVI;

b) por razones históricas que siguen despertando polémica, en una gran parte del continente americano dicho retroceso sucede más tarde, es decir, hacia el siglo XVIII y, por tanto, cantare todavía se utiliza en el español atlántico del siglo XX;

c) debido al empleo reducido del antefuturo de subjuntivo a lo largo de toda su historia, es más difícil determinar el momento en el que ese tiempo queda eliminado de la lengua. Sin embargo, a la luz de los datos recopilados en el CORDE, creemos que cae en desuso en España hacia el siglo XVIII. Teniendo en cuenta la cronología de la desaparición de cantare, se supone que hubiere cantado pierde la vitalidad un poco más tarde en América que en la Península, aunque en este caso también pueden darse diferencias en distintas áreas.

Nos damos cuenta de que este pequeño análisis no es del todo exhaustivo y de que hay una serie de problemas relacionados con la eliminación de hubiere cantado que quedan por resolver. Sin embargo, debido a la dimensión restringida del presente tomo, nos vemos obligados a dejar estas cuestiones, esperando que sean estudiadas con mayor detenimiento en los momentos venideros más oportunos.

\section{Bibliografía}

CAMÚS BERGARECHE, B. (1990). «El futuro de subjuntivo en español», en I. BOSQUE (ed.), Indicativo y subjuntivo. Madrid: Taurus Universitaria, 410427.

CORDE - REAL ACADEMIA ESPAÑOLA: Banco de datos (CORDE) [en línea] $<$ http://www.rae.es>, fecha de consulta: 4 de septiembre de 2018.

EBERENZ, R. (1990). «Sea como fuere. En torno a la historia del futuro de subjuntivo en español», en I. BOSQUE (ed.), Indicativo y subjuntivo. Madrid: Taurus Universitaria, 383-409.

FOLGAR, C. y RAMOS, M. (1992). «Privilegios de figuración del futuro de subjuntivo en español medieval», en M. ARIZA et al. (eds.), Actas del II Congreso Internacional de Historia de la Lengua Española, vol. I. Madrid: Pabellón de España, 403-427.

FONTANELLA DE WEINBERG, M. B. (1987). El español bonaerense. Cuatro siglos de evolución lingüística (1580-1980). Buenos Aires: Hachette. 
FONTANELLA DE WEINBERG, M. B. (1992). «Historia del español de Argentina», en C. HERNÁNDEZ ALONSO (ed.), Historia y presente del español de América. Valladolid: Junta de Castilla y León, 357-382.

GRANDA DE, G. (1978). «Formas en -re en español atlántico y problemas conexos», en Estudios lingüísticos hispánicos, afrohispanos y criollos. Madrid: Gredos, 95-117.

HERRERO RUIZ DE LOIZAGA, F. J. (2006). «Cronología y usos del futuro de subjuntivo», en M. VILLAYANDRE LLAMAZARES (ed.), Actas del XXXV Simposio Internacional de la Sociedad Española de Lingüística. León: Universidad de León [en línea] <http://fhyc.unileon.es/SEL/actas.htm>, fecha de consulta: 6 de septiembre de 2018, 940-956.

LUQUET, G. (1988). «Sobre la desaparición del futuro de subjuntivo en la lengua hablada de principios del siglo XVI», en M. ARIZA, A. SALVADOR y A. VIUDAS (eds.), Actas del I Congreso Internacional de Historia de la Lengua Española, vol. I. Madrid: Arco / Libros, 509-514.

LÓPEZ RIVERA, J. J. (1994). El futuro de subjuntivo en castellano medieval. Santiago de Compostela: Universidade de Santiago de Compostela (Verba, anexo 38).

NGLE - Real Academia Española (2009). Nueva gramática de la lengua española. Madrid: Espasa Calpe.

NOWIKOW, W. (1993). Evolución funcional de los esquemas condicionales no reales en el español de los Siglos de Oro. Łódź / Frankfurt: Wydawnictwo Uniwersytetu Łódzkiego / Vervuert Verlag.

NOWIKOW, W. (1995). «Sobre las causas de la eliminación del futuro de subjuntivo del sistema verbal español», en A. BOCHNAKOWA y S. WIDŁAK (eds.), Munus Amicitiae. Studia Linguistica in Honorem Witoldi Mańczak Septuagenarii. Kraków: Uniwersytet Jagielloński, 111-117.

PENNY, R. (2014). Gramática histórica del español. Barcelona: Ariel.

RAMÍREZ LUENGO, J. L. (2002). «El futuro de subjuntivo en la Banda Oriental del siglo XVIII», Revista de Filología, 20, 305-317.

RAMÍREZ LUENGO, J. L. (2008). «El futuro de subjuntivo en el español centroamericano del siglo XVIII. Vitalidad, empleo e indicios de decadencia», Nueva Revista de Filología Hispánica, LVI (1), 141-154.

ROJO, G. y VEIGA, A. (1999). «El tiempo verbal. Los tiempos simples», en I. BOSQUE y V. DEMONTE (eds.), Gramática descriptiva de la lengua española, vol. 2. Madrid: Espasa-Calpe, 2868-2934. 